\title{
Drift Compression Experiments on MBE-4 and Related Emittance Growth Phenomena*
}

S. Eylon, A. Faltens, W. Fawley, T. Garvey,

K. Hahn, E. Henestroza and L. Smith

Laurrence Berkeley Laboraiery,

University of California

Berkeley, CA 94720

April 1991

This report has been reproduced directly from the best available copy.

* Work supported by the Director, Office of Energy Research, Office of Basic Energy Sciences, Advanced Energy Projects Division, U.S. Department of Energy under Contract No. DE-AC03-76SF00098. 


\title{
Drift Compression Experiments on MBE-4 and Related Emittance Growth Phenomena*
}

\author{
S. Eylon, A. Faltens, W. Fawley, T. Garvey, K. Hahn, E. Henestroza, and L. Smith, \\ Lawrence Berkeley Laboratory, 47/112 \\ Usiversity of California \\ Berkeley, CA 94720 USA
}

\section{Abstract}

We have recently conducted a series of experiments on the MBE-4 heavy ion accelerator in which a velocity tilt was placed on the beam in the first accelerating section beyond the injector, followed by drift compression over the remaining 11 meters. Depending upon the magnitude of the velocity tilt and the accompanying mismatch in the focusing lattice, emittance growth was observed, manifested by "butterfly" shapes in $x-x^{\prime}$ phase space. We discuss various analycical limits on ion beam compression and relate tbem to these experiments and also to a driver for a heavy ion fusion reactor. We also present numerical simulations which investigate various aspects of compression and consequent emittance growth.

\section{Incroduction}

MBE-4 is a multiple beam, heavy ion accelerator at LBL desigmed to study the phyoics of space-charge dominated beans and scaling thereof to a heavy ion fusion "driver". One of the major requirenients for a driver is smooth and controlled compression of the heavy ion besm from a typical duration at the injector $(E \sim 2-10 \mathrm{MeV})$ of $\sim 10$ / $\mathrm{sec}$ to a duration at accelerator exit $(E \sim 5-10 \mathrm{GeV})$ of $\sim 100$ ns. In general, this temporal compression will involve a smaller, but not insignificant increase in line charge density also. There is an additional $\sim 10 \mathrm{x}$ compreasion at nearly constant energy between the accelerator and target to bring the pulse duration to the wanted $10 \mathrm{~ns}$.

In the last year, we have conducted a number of compression experiments on MBE-4 both at contant energy (i.e. drift compression) and with steady acceleration [1] from the injector energy of $185 \mathrm{kV}$ to final energie of $\sim 800$ kV. In both cases, the compression is achieved via a headto-tail velocity tilt on the beam. Of particular concern is the behavior of the ion bearn transverse phase space during the longitudinal compression. Our results suggest that very strong compression ratios $(\geq 6: 1$ ) lead to substantial emittance growth for MBE-4 besms while smallet ratios ( $\leq 3: 1)$ generally cause little or no emittance growth. As explained in SIV, the key physics appears to be the interaction between a highly compressed and thus radially large beam and the external nonlinear dodecapole focusing forces and internal, nonlinear space charge fields.

\footnotetext{
- Work supported by the Director, Office of Energy Revearch, Otfice of Basic Enerby Sciencen, Advanced Eneror Projecte Division, U.S. Depl. of Energy under Contrace No. DE-AC03-76SF00098.
}

\section{Experimental Set-up}

For the drifl compresion experiments described here, MBE-A was used in a single beam mode with the 185kV injector producing a 2.5- $\mu$ duration, 8-mA current pulse of $\mathrm{Cs}^{+1}$. As the $\mathrm{t}$ aam leaves the injector, it passes through a "matching zone" composed of a eight individual quadrupoles used for transition onto the syncopated FODo focusing lattice of the main accelerator. Within this matching zone, there is an aperture plate that absorbs the outer (and most badly aberrated) portions of the beam, reducing the current by a factor of two. In general, the matching is imperfect and the beam's radial profile shows low level hollowing oscillacions between lattice period 0 $(\equiv L P 0)$ and LP10. The electrostatic quadrupole voltages were set to produce a single particle phase advance $\sigma_{0}$ of 72 per lattice period; the space-charge depressed tune $\sigma$ was in the range $7-10^{\circ}$.

The first four accelerating gaps were timed so that a nearly linear velocity tilt (ranging from 0 to $\geq 12 \%$ ) was put on the heam. We used a numerical code SLIDE[1] to determine the timing and amplitude of the accelerabing pulses. No further acceleration fields were applied downstream and thus the beam energy remained constant apart from the work done against the longitudinal space charge field. An energy analyzer (normally positioned at LP5) measured the temporal variation of the beam's en. ergy with a. $0.5 \%$ energy and $\leq 20$-ns temporal resolution. At every five lattice points along the accelerator, a two-slit emiltance scanner determined the $x-x^{\prime}$ projection of the beam's tranverse phase space. In general, the $z$ resolution of $\approx 1.0 \mathrm{~mm}$ was mueh smaller than the typical beam radius of 5-10 mm, while the angular resolution of $0.7 \mathrm{mrad}$ was comparable to the projected RMS wridth. The emittance scans were prograrimed to trare out a parallelogram in phase space with a tilt equal to that corresponding to the temporal center of the beam's phase space ellipsoid.

\section{Results}

Our initial drift compression experiments were with a quite vigorous acceleration schedule which resulted in a nearly $5: 1$ current increase by LP15, and $7.4: 1$ by LP20. The RMS emittance also tripled and butterfly shapes were evident in the phase space data We then ran a number of less vigorous compression schedules to determine the sensitivity and behavior of the emittance increase. 
Table 1. Emittance $(\varepsilon)$ and beam radius $(a)$ measurements for drift compresion experiments

\begin{tabular}{|c|c|c|c|l|}
\hline \hline$I_{\text {max }} / I_{0}$ & $\varepsilon_{\max }^{r \max } / \varepsilon_{0}^{r m a}$ & $a_{\operatorname{mex}} / a_{0}$ & $\varepsilon_{\max }^{\mu} / \varepsilon_{0}^{\mu}$ & $N_{\max }$ \\
\hline $1: 1$ & $0.9-1.1$ & $1.0-1.2$ & $1.1-1.2$ & - \\
\hline $1.5: 1$ & $0.9-1.1$ & $0.9-1.0$ & 1.0 & $\geq \mathrm{LP20}$ \\
\hline $3.7: 1$ & $1.3-1.6$ & 1.6 & $1.0-1.2$ & LP25 \\
\hline $7.4: 1$ & $\geq 2.8-3.2$ & $\geq 1.7-2.0$ & $\geq 2.1$ & LP20 \\
\hline \hline
\end{tabular}

Table 1 presents various measured quantities summarixing the results of the different compression schedules. The "microscopic" emittance $\varepsilon^{\mu}$ is defined to be the phase space area (not necessarily contiguous) occupied by the moat intense $80 \%$ fraction of the beam current. This quantity is readily extracted from the $2 \mathrm{D}$ map produced by the emittance scanner. $\epsilon^{\mu}$ is expected to be a more "conserved" quantity than $\varepsilon^{r m}$ in cases such as a simple " $S$ " distortion of the $x-z^{\prime}$ phase space. $N_{\operatorname{mar}}$ is the lattice period at which maximum compression was measured. For the 7.4:1 compression data, it appears that portions of the heam extended outside the maximum range scanned in $\boldsymbol{x}$, $\pm 20 \mathrm{~mm}$ (the clear quadrupole aperture is $27.4 \mathrm{~mm}$ ).

In Fig. 1 we plot $\varepsilon(z)$ for various longitudinal slices of the bearn pulse in the 3.7:1 compression experiment. The slices are labeled by their charge-weighted positions in the beam with the presumption that no longitudinal overtaking has occurred. Two observations are of note: 1. Slices with little compression $\left(I / I_{0} \leq 2.7\right)$ suffered lit tle emittance growth and those in free expansion near the head and tail may actually have "cooled off' with increasing $z$. 2. The mid-pulse slices with significant emittance growth by LP25 showed little grownh at earlier positions in $z$ where the compression was $\leq \mathbf{3 . 0}$.

This first observation is aleo true for the $7.4: 1 \mathrm{com}$ pression data alchough the sparsity of observation points (LPO-LP20 only) prevents one from drawing firm conclusions concerning the final state of the beam. Moreover, both high compression cases show that the "microscopic" transverse phase space areas of the betam increased far less than did the RMS measures. For the 3.7:1 care, the relative increase in $\varepsilon^{\mu}$ is essentially unchanged from that measured for the simple 1:1 drift case. Figure 2, which plots phase space contours of a central bearn slice at LP25, shows why this is so: the phase space ellipse has developed " $S^{n}$-arms which account for the increase in RMS emittance but do not result in an actual phase space dilution. By contrast, Fig. 3, which plots phase space contours for the central slice of the $7.4: 1$ compression case, sbows a "butterfy" or bow tie shape in its wings. The butterfly wings include both a major portion ( $\geq 30 \%$ ) of the current at a given instant in time, and persist for the great majority of longitudinal slices measured at LP20. In this case, $x-x^{\prime}$ phase space has been truly diluted and no simple system of lenses will reverse this degradation.

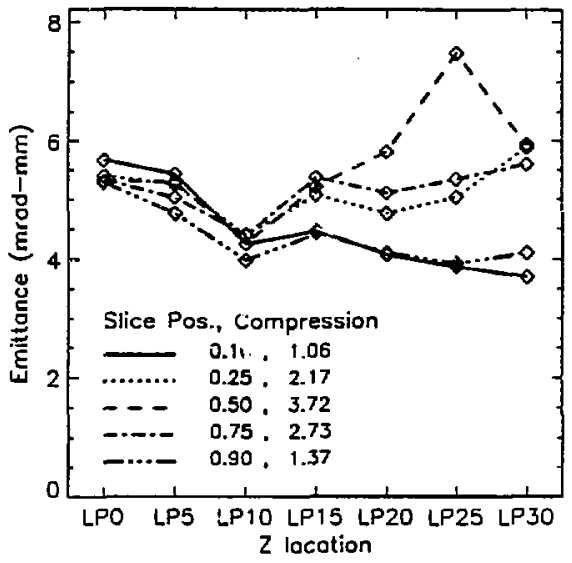

Figure 1: RMS emittance measturements for various longitudinal slices in the 3.7:1 drift compression data. The first number refers to the chargeweighted position in the beam (i.e. head $=0$, mid-pulse $=0.5$, tail $=1$ ) while the second refers to the maximum compression measured for a given longitudinal slice.

\section{Analysis and Discussion}

After discovering the butterfly shapes in the high compression phase space data, some of us suspected that longitudinal overtaking might be oceurring within the beam near the LP15-LP20 region. Supporting this view was an obseryed rapid temporal change in the phase space ellipsoid tilts, exceeding $90^{\circ}$ in a time half that of the curreat pulse FWHM. Perhaps the butterfly shapes were actually the superposition of two beamlets of different energies whose integrated phase advance differed by $\sim 90^{\circ}$. Bowever, the SIIDE numerical sode, which has been quite successful in predieting current waveforms for MBE-4 compressed beams, did not suggest problems with our-i aking. Furchermcre, energy analyzer scang taken at LPI5 and LP20 do not show obvious double-yalued behavior that would be a sign of longitudinal overtaking.

A related possibility is that while no longitudinal overtaking per se occurred, the longitudinal bean compression might bave become so great in tive 7.4:1 case that the lon. gitudinal variation in the $z$-integrated phase advance tor mismatch oscillations has become significant ( $\geq$ ! cadian) over a quadrupole aperture radius $b$. If so, this allows "communication" via space charge fields between slices of different mismatch phase and might lead to relatively rapid phase mixing. For our case of $\sigma \ll \sigma_{0}$, we estimate the 


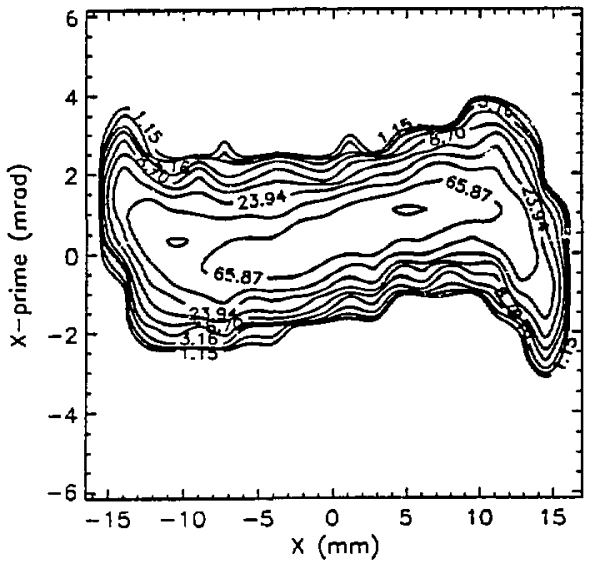

Figure 2: Phase space density contours (equi-spaced logarithmically) measured at LP25 for the central loogitudinal slice of the 3.7:1 drift compression data.

maximum phase advance variation as

$$
\Delta_{\phi}=\int_{L P S}^{L P 30} \sqrt{2} \sigma_{0} d N\left|\frac{d \rho}{\beta d !}\right| \frac{b}{\beta_{0} c} \approx 0.6 \text { radians } .
$$

Here $N$ measures $z$ in lattice periods. Given the relatively rapid (in $z$ ) reversal of the beam's compression which limits the communication time, it is unclear whether this effect and the magnitude of mismatch oscillations are sufficient to produce transverse phase space shapes such as Fig. 3.

A second suggested mechanism for emittance growth was the interaction of a compressed (and thus "fat") beam with the small $(\approx 10 \%$ relative to the qusdrupole at the electrode surface) nonlinear dodecapole forces present in the MBE-4 lattice. Previous experiments [2] on MBE4 bave shown that dodecapole-induced phase-mixing for off-axis beams can lead to significant emittance gromth. In the present experiments, however, the beam remained within $\approx \pm 0.5 \mathrm{~mm}$ of the axis, so simple centroid damping is ruled out. Particle simulation code runs [2] suggest that dodecapole-induced emittance growth will occur for centered, drift-compressed besms with the predicted magnitude sufficient to explain the 3.7:1 compression data. The simulation results are not as clear-cut for the 7.4:1 case, due to difficulties such as partiele loss to the walls (which possibly occurred in the experiment also). D. Grote at LLNL (private communication), however, has produced butterfly shapes in a 3D simulation of MBE4 compression including dodecapole forces. Space charge non-uniformities, which appear to he quite importane for

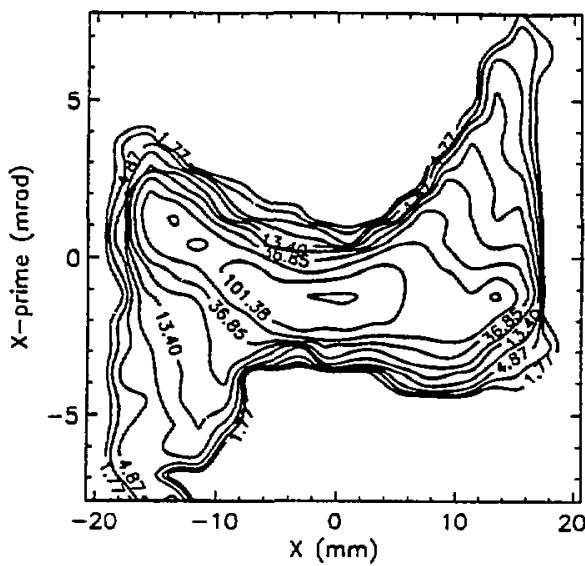

Figure 3: Phase space density contours measured at LP20 for the central longitudinal slice of the 7.4:1 drift compression data

$z \geq L P 10$ in the 7.4:1 data, probably also affected the evolution of the transverse phase space. Such non-uniformities (both symmetric and non-symmetric) can present strong. nonlinear perturbations for highly space-charged depressed beams such as MBE-4.

Summarizing, strong (7.4:1) and rapid compression $\left(d \lambda / \sigma_{0} d N \geq 0.1 \lambda\right)$ of the MBE-4 beam led to large emittance growth. We believe that the cause is interaction of the outer portions of the beam with nonlinear dodecapole focusing forces and possibly non-uniform space charge distributions. Weaker (3.7:1) and less rapid compression $\left(d \lambda / \sigma_{0} d N \leq 0.05 \lambda\right.$ ) showed little or no RMS emittance growth. Most MIF driver designs have $d \lambda / \sigma, d N \leq 0.005 \lambda$ within the accelerator. Hence, non-adisbatic, rapid compression problems, if they oceur at all, are far more likely in the final focus section ratber than within the accelerator.

\section{References}

[1] T. Garvey et. al, "Transverse Emittance Studies of an Induction Accelerntor of Heavy lous", Poster XRA51, the se proceedings.

[2] S. Eylon et al., "Emitance Variations of Very Cold Icn Bearns During Transport Through MBE-A", in Proc. Int. Symp. on Beavy lon Inertial Fusion, Monterey, CA, Dec., 1990, to appear in Part. Accel., 1991. 